\title{
Acoustic analysis of snoring before and after palatal surgery
}

\author{
T.M. Jones*, A.C. Swift*, P.M.A. Calverley*, M.S. Ho" and J.E. Earis*,
}

\begin{abstract}
To investigate the effectiveness of palatal surgery for nonapnoeic snoring, 35 patients were block randomised to undergo one of two different palatoplasty procedures. Patients were admitted pre-operatively for audio recording of snoring sound and video recording of sleeping position, and between 1.0 and 4.1 months (mean 2.5) and between 5.9 and 17.5 months (mean 9.7) post-operatively. Sound files, comprising the inspiratory sound of the first 100 snores whilst sleeping in a supine position, were analysed using specifically designed software.

Snore duration (s), loudness (dBA), periodicity (\%) and energy ratios for the frequency bands 0-200 Hz, 0-250 Hz and 0-400 Hz were calculated. Subjective outcomes were noted. Operation type, body mass index, age, peak nasal inspiratory flow rate, Epworth sleep score and alcohol intake were considered as confounding variables.

No patient was cured from snoring. Paired t-test analysis demonstrated statistically significant changes between pre- and early post-operative recordings for snore periodicity and energy ratios in the frequency ranges $0-200 \mathrm{~Hz}, 0-250 \mathrm{~Hz}$ and 0-400 Hz.

In conclusion, only the $0-250-\mathrm{Hz}$ energy ratio measurements maintained a statistically significant improvement at the time of the late post-operative recording, despite an obvious drift back to pre-operative levels. No confounding variables were identified. The subjective and objective results correlated poorly. Post-operative changes in the acoustic parameters of snoring sound, following palatal surgery, are demonstrable but short-lived.
\end{abstract}

KEYWORDS: Acoustic analysis, palatoplasty, sleep, snoring

oud snoring, present on most or every night, is reported by $24-50 \%$ of males [1] and $14-30 \%$ of females $[1,2]$. This may cause social embarrassment, contribute to relationship disharmony and marriage breakdown [3]. In addition, heavy snoring, even without sleep apnoea, has been implicated in the aetiology of hypertension, ischaemic heart disease, cerebrovascular accidents, and increased morbidity and mortality from road traffic and workrelated accidents. Factors precipitating snoring include nasal obstruction, upper airway morphology (especially the degree of micrognathia), obesity (characterised by large collar size and high body mass index (BMI)), and excess alcohol and cigarette consumption $[4,5]$.

Although snoring can arise from the vibration of many structures in the upper airway, the soft palate is considered to be the major sound generator [6-8]. Based on this assumption, various types of palatal surgical techniques have been developed. Initially, uvulopalatopharyngoplasty (UPPP), a procedure involving the removal of up to $2 \mathrm{~cm}$ of the soft palate, together with tonsillectomy and suture apposition of the faucial pillars, was introduced for patients with sleep apnoea in 1964 by IKEMATSU [9], and later popularised by FuJITA et al. [10]. Subsequent reports suggested that UPPP was not an effective treatment for sleep apnoea, but did reduce snoring [11, 12]. However, as UPPP is associated with a high complication rate (nasal regurgitation, nasopharyngeal stenosis, hypernasal voice and even death) $[4,13]$, less extensive procedures designed to limit soft palate movement, either by reducing its length or stiffening its tissues, were devised. In the current study, two such procedures were used, traditional palatoplasty with uvulectomy (TP), as described in the early 1990s by ELLIS et al. [14], and uvulopalatal elevation palatoplasty (UEP) [15].

Acoustic analysis of snoring sound has identified two main types of snoring waveform: 1) a complex waveform of low-frequency sound, generated as a result of collision of opposing airway walls during periods of brief airway closure, typical of palatal flutter; and 2) a simple waveform sound with a quasi-sinusoidal pattern thought to result from oscillations of the airway walls around a neutral position without closure
AFFILIATIONS

${ }^{\star}$ Clinical Science Centre, and

-Aintree Chest Centre, University

Hospital Aintree, Liverpool, and

\#Dept of Computer Science,

University of Manchester,

Manchester, UK.

CORRESPONDENCE

T.M. Jones

Dept of Otolaryngology

Head and Neck Surgery

University Hospital Aintree

Long Lane

Liverpool L9 7AL

UK

Fax: 441515295263

E-mail: terryjones@doctors.org.uk

Received:

September 092004

Accepted after revision:

January 262005

European Respiratory Journal

Print ISSN 0903-1936

Online ISSN 1399-3003 
of the airway lumen [6]. To date, there are few rigorous, randomised, controlled clinical trials relating to snoring surgery. Of those that do exist, none have utilised acoustic changes of snoring sound as objective outcome measurements [16].

It was hypothesised that the physical characteristics of the upper airway would determine the acoustic waveform and that surgical therapy would modify these. To investigate this, a longitudinal study of the effects of upper airway surgery on the acoustic characteristics of snoring was conducted. A randomised controlled design was adopted to determine the effects of two different surgical treatments, and the objective indices of snoring and the patients' subjective reports of symptoms were compared.

\section{PATIENTS AND METHODS Subjects}

In total, 54 patients awaiting admission to hospital for palatal surgery were enrolled. All complained of loud snoring every night, which caused social disruption. Patients were screened either with overnight polysomnography or limited respiratory sleep studies to exclude unsuspected obstructive sleep apnoea. Patients with an apnoea hypopnoea index (AHI) of $>15 \cdot \mathrm{h}^{-1}$ were excluded from the study. All patients snored heavily in the supine position. All gave informed consent and ethical approval was obtained.

\section{Procedures}

TP was performed transorally on an anaesthetised patient. Using a $\mathrm{CO}_{2}$ laser, a central strip of soft palate mucosa, extending from the junction of the hard and soft palate to the free edge of the soft palate, was excised. The uvula was then amputated [17]. It is hypothesised that fibrosis resulting from tissue healing results in a stiffening of the soft palate, a change in its aerodynamic properties and, consequently, a decrease in the probability of soft palate flutter in the airflow generated by inspiration [17].

UEP involved the removal of a central strip of soft palate mucosa as described previously. However, in this case, excision of the soft palate musosa extended onto the oral surface of the uvula. Instead of amputating the uvula, as in the TP, the raw surfaces of the soft palate and uvula were brought into apposition by reflecting, and then suturing, the oral surface of the uvula onto the oral surface of the soft palate. The reflection of the uvula was facilitated by lateral palatal-releasing incisions. This resulted in elevation, shortening, thickening and stiffening of the soft palate and, as with TP, this is believed to reduce soft palate flutter in the inspiratory airflow [15].

\section{Protocol}

Initial assessment included a full clinical history, physical examination, calculation of BMI $\left(\mathrm{kg} \cdot \mathrm{m}^{-2}\right)$ and the best of three maximum peak nasal inspiratory flow rate (PNIFR) readings using a Youlten, Airmed ${ }^{\mathrm{TM}}$ Peak Nasal Inspiratory Flow meter (Clement Clarke International Ltd, Harlow, UK).

Patients were block randomised into two groups to undergo UEP $(n=28)$ or TP including uvulectomy $(n=26)[14,15]$. Each group was stratified according to a BMI of $>30$ or $<30 \mathrm{~kg} \cdot \mathrm{m}^{-2}$. All surgery was performed by the same surgeon.
Patients were admitted overnight to a specifically designated quiet single room and acoustic measurements were made on the evening preceding scheduled surgery, and then $\sim 2$ and 9 months after surgery.

\section{Acoustic and video measurements}

Snoring sounds were recorded onto a Sony digital audio tape (DAT) recorder (TCD-D8; Sony United Kingdom Ltd, Newbury, UK) using a microphone suspended from the boom arm of a microphone stand, $60 \mathrm{~cm}$ above the mattress at the head end of the bed. An infrared camera, linked to a domestic video recorder, was directed at the patient's head.

Using a sound-level meter (Cirrus ${ }^{\mathrm{TM}}$; Cirrus Research plc, Hunmanby, UK) positioned at the level of the recording microphone, a loudness calibration curve was created by recording and measuring the sound level, in $\mathrm{dBA}$, of simulated snores produced by a volunteer lying supine on the bed. Direct comparison of the amplitudes of the recorded natural snores with the calibration curve allowed calculation of the loudness of the snoring.

On retiring, patients were instructed to switch on both audio and video recorders. Both recorders automatically switched off when the tape space had been fully utilised.

At the time of their late post-operative recording, patients answered a specifically designed questionnaire comprising of six distinct categories relating to demographic data, the operation, subsequent sleep quality, swallowing and voice, pharyngeal sensation and snoring.

\section{Acoustic and video analysis}

The 4-h DAT recordings (sampling rate of $8,000 \mathrm{~Hz}$ at 16-bit resolution) were directly downloaded onto the computer hard disc via an optical linkage, using a specifically designed adapter board (Pocab ${ }^{\mathrm{TM}}$; Designated Associates Ltd, Birkenhead, UK). Using the video recordings and the audio software package Cool Edit ${ }^{\mathrm{TM}}$ (Adobe Systems, Uxbridge, UK), the recordings were manually searched for typical snoring sounds. Following acoustic identification of a presumed snore, the video tape was scrutinised at the corresponding point in time to establish, first, whether the patient appeared to be asleep and, secondly, whether they were sleeping in a supine position. In this way, sound files comprising the first 100 inspiratory snores with the patient sleeping in a supine position were created and used for all the subsequent analyses. A specifically developed software programme formatted within the Matlab ${ }^{\mathrm{TM}}$ software package (The Mathworks Ltd, Cambridge, UK) was used to calculate the following acoustic parameters from these snore files. 1) Snore duration (s): the average duration of each inspiratory snoring sound. 2) Snore loudness (dBA): the loudness calibration curve was used to calculate the loudness of each individual snore. The average loudness of the inspiratory snoring sounds included in the 100 snore files was also calculated. 3) Snore periodicity (\%): snore periodicity was measured by segmenting the 100 snore files of each patient into 200-ms frames. Each frame was classified as either periodic or nonperiodic, based on the autocorrelation function of the frame [18]. Snore periodicity is defined as the ratio of the number of periodic frames to the total number of frames. 4) Energy ratio: previous studies have characterised 
palatal snores as low-frequency sound [19-21]. Therefore, using a 1,024-point fast fourier transform (FFT), the energy content of the frequency sub-bands $0-200 \mathrm{~Hz}, 0-250 \mathrm{~Hz}$ and $0-$ $400 \mathrm{~Hz}$ was calculated on a frame-by-frame basis at an update interval of $100 \mathrm{~ms}$. The total energy of the 100 snore files was similarly calculated. The frequency-band energy ratio for the 100 snore files is defined as the ratio of the sub-band energy, to the total energy of all frames.

Of the original 54 patients recruited for the study, 19 were excluded.

\section{Statistical analysis}

A repeated-measures ANOVA analysis was performed for the pre-operative, early post-operative (post-op 1) and late postoperative (post-op 2) acoustic measurements. Pre-operative BMI ( $>30$ or $<30$ ), type of palatoplasty performed and patient sex were incorporated as between-grouping variables. Subsequently, paired t-test analysis, incorporating a Bonferroni correction, was performed to compare the preoperative results with early and late post-operative results. Multiple regression analysis, including forward stepwise regression analysis, was used to test for statistically significant contributions of age, PNIFR, Epworth sleep scale results or alcohol intake (units $\cdot$ week $^{-1}$ ) to the surgical outcome. The questionnaire responses were of a forced-choice format, scored using a Likert scale and analysed using nonparametric statistical methods. Spearman rank correlation analysis was used to compare subjective and objective outcomes.

\section{RESULTS}

Of the 54 patients, 11 withdrew pre-operatively, two failed to attend for early and five for late follow-up measurements, and one was subsequently found to have a pre-surgical AHI $>15 \cdot h^{-1}$ and was excluded from the final analysis. The demographic data of the 35 patients who completed the trial, divided by their surgical treatment, did not differ (table 1). All pre-operative data were obtained the day before surgery. The first post-operative recordings were made between 1.0 and 4.1 months (mean 2.5 months), whilst the second post-operative

\begin{tabular}{lcc}
\hline TABLE 1 & $\begin{array}{l}\text { Comparison of demographic data between the } \\
\text { uvulopalatal elevation palatoplasty (UEP) group } \\
\text { and the traditional palatoplasty (TP) group }\end{array}$ \\
Variable & UEP & TP \\
\hline Subjects $\mathbf{n}$ & 19 & 16 \\
Males/females & $17 / 2$ & $13 / 3$ \\
Mean age yrs & $47(33-60)$ & $46(24-65)$ \\
Mean BMI kg $\mathbf{m}^{-2}$ & $31.4(24.9-47.2)$ & $29.6(24.3-36.0)$ \\
Mean PNIFR & $148(90-230)$ & $135(80-190)$ \\
Median EtOH units $\cdot$ week $^{-1}$ & $4(1-6)$ & $3(1-7)$ \\
Median ESS & $7(0-15)$ & $9(2-17)$ \\
Median AHI & $2.7(0-12.8)$ & $3.8(1.0-10.9)$ \\
\hline
\end{tabular}

Data are presented as mean (range) or median (range), unless otherwise stated. BMI: body mass index; PNIFR: peak nasal inspiratory flow rate; EtOH: ethyl alcohol; ESS: Epworth sleep scale; AHI: apnoea-hypopnoea index. No statistical difference is demonstrable, for all parameters, between the two groups. recording occurred between 5.9 and 17.5 months (mean 9.7 months) after surgery.

\section{Acoustic analysis}

Two-way, repeated-measures ANOVA of the pooled data for all patients demonstrated statistically significant differences between pre- and post-operative measurements for loudness, periodicity and the energy ratios for each frequency sub-band. However, there were no statistically significant differences between the sub-group analysis for different operations, BMI values and sex.

Compared with the pre-operative data, the early post-operative results showed significant improvements in snore periodicity and $0-200,0-250$ and $0-400-\mathrm{Hz}$ energy ratios $(\mathrm{p}<0.001,0.002$, 0.002 and 0.047 , respectively). This difference was lost when pre-operative and late post-operative results were compared, although a marginally significant difference in the $0-250-\mathrm{Hz}$ energy ratio measurements was still present $(\mathrm{p}=0.039)$, despite a clear drift back to pre-operative levels (fig. 1).

Although snore loudness fell between the pre-operative and early post-operative recordings, this change did not reach statistical significance, nor was there any difference between the pre-operative and late post-operative recordings. However, paired comparison of snore loudness between the early and late post-operative recordings showed a significant increase in snore loudness between recordings $(p<0.001)$.

Age, PNIFR, Epworth sleep score and reported alcohol intake (units $\cdot$ week $^{-1}$ ) were unrelated to either the early improvement or subsequent decline in the acoustic parameters after surgery.

A further analysis of the statistically significant variables (periodicity, $0-200-\mathrm{Hz}$ and $0-250-\mathrm{Hz}$ energy ratios) for the consistency of these changes between visits in individual patients is presented in table 2 . In only 12 cases $(34.3 \%)$ for periodicity, nine $(25.7 \%)$ for the $0-200-\mathrm{Hz}$ band and $11(31.4 \%)$ for the $0-250-\mathrm{Hz}$ band were the second post-operative values the same as, or lower than, the first post-operative values. Therefore, depending on the acoustic measurement chosen, between a quarter and a third of patients appeared to show a sustained improvement in these acoustic variables at the late post-operative recording.

\section{Questionnaire}

The questionnaire was completed by 34 patients at a median (range) of 21 months (11-26) after surgery. Among the respondents, four out of $34(11.8 \%)$ reported no post-operative improvement, 19 out of $34(55.9 \%)$ reported an initial improvement that was not sustained for longer than $1 \mathrm{yr}$, whilst 11 out of $34(32.3 \%)$ reported an improvement "until now" (ranging 19-26 months with a median of 21 months).

These results are of a similar magnitude to the improvements in the acoustic measurements; however, there was no relationship between the objective acoustic and subjective questionnaire outcomes on an individual patient level.

\section{DISCUSSION}

The relative accessibility of the upper airway has made it an attractive target for surgical interventions that are designed to abolish both obstructive sleep apnoea and snoring. However, 

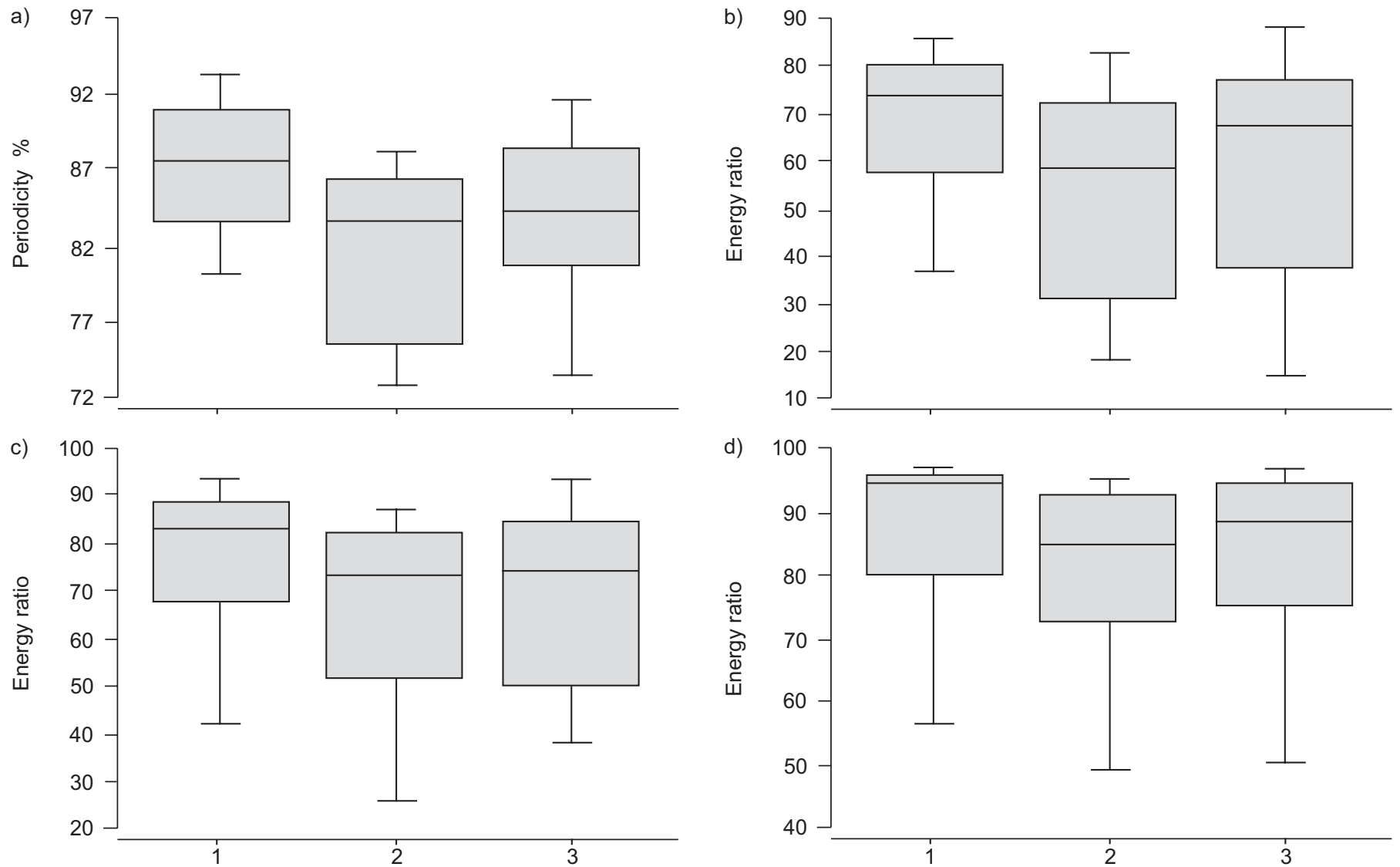

FIGURE 1. Box and whisker plots representing the 10th, 25th, 50th, 75 th and 90th centiles of the data distribution for a) snore periodicity, b) snore energy ratio: 0 $200 \mathrm{~Hz}, \mathrm{c}$ ) snore energy ratio: 0-250 Hz, and d) snore energy ratio: 0-400 Hz. Plots 1, 2 and 3 relate to pre-operative, first post-operative and final post-operative visits, respectively. $p$-Values for the overall change $(p)$, pre-operative to post-operative visit one $(p 1,2)$ and pre-operative to post-operative visit two (p1,3), are as follows: a) $p<0.001, p 1,2<0.001, p 1,3=0.079$ (NS); b) $p=0.017, p 1,2=0.02, p 1,3=0.077$ (NS); c) $p=0.002, p 1,2=0.002, p 1,3=0.039 ;$ and d) $p=0.025, p 1,2=0.047, p 1,3=0.126$ (NS)

\section{TABLE 2 Individual surgical outcome}

\begin{tabular}{lccc} 
Outcome & $\begin{array}{c}\text { No } \\
\text { improvement }\end{array}$ & $\begin{array}{c}\text { Initial improvement } \\
\text { not sustained }\end{array}$ & $\begin{array}{c}\text { Sustained } \\
\text { improvement }\end{array}$ \\
\hline Snore periodicity & $5(14.3)$ & $10(28.6)$ & $20(57.1)$ \\
Energy ratio $\mathbf{0 - 2 0 0 ~ H z ~}$ & $8(22.9)$ & $6(17.1)$ & $21(60.0)$ \\
Energy ratio $\mathbf{0 - 2 5 0 ~ H z}$ & $5(14.3)$ & $7(20.0)$ & $23(65.7)$ \\
\hline & & & \\
Data are presented as $\mathrm{n}(\%) . \mathrm{n}=35$.
\end{tabular}

the history of surgery for these related problems has followed a similar course, with initial enthusiasm and significant "cure" rates being followed by disappointment as significant relapse rates are identified when follow-up is extended beyond the first few months of surgery. Thus, upper airway surgery directed at the soft palate is now recognised as being of little value in most patients with obstructive sleep apnoea, as it does not address the primary anatomical and physiological reasons for the disorder. The present data suggest that the same is likely to be true for patients without frequent apnoeic events who present with socially disruptive snoring.
Unlike previous, more positive reports, the current authors used objective acoustic measurements to determine the effect of surgery. Subjective measures are notoriously difficult to validate as they depend on the patient and their partner's perception of snoring intensity, frequency and the quality of the snoring sound. Subjective assessment is likely to be even more unreliable after the patient has undergone painful palatal surgery to try to please their partner and restore social harmony. In contrast, acoustic analysis is objective and observer independent. As in many other studies, the present authors saw little relationship between objective and subjective measurements [22-24], suggesting that factors other than the acoustic properties or frequency of the snore determine patient satisfaction with these procedures.

Snoring occurs when transient airway collapse develops due to inspiratory flow limitation. However, the actual sound may be generated at sites distant from the flow-limiting segment. Potential sites of snoring sound include the vibration of upstream structures (e.g. soft palate), oscillations of the airway walls at the point of maximal collapse and the turbulent nature of airflow itself $[6,25]$. Although the soft palate is assumed to be the primary noise generator, other structures of the supraglottis or oropharynx, as well as tongue base and 
epiglottis, may also vibrate to a greater or lesser extent in any one individual [8]. In these circumstances, it is no surprise that the present authors found that treatment directed at the soft palate did not abolish snoring in any of the patients, even temporarily.

Surgery did modify the acoustic characteristics of the snoring, at least temporarily. Vibration of the soft palate is believed to generate a relatively low-frequency sound [19-21], and both surgical procedures reduced the energy content of the lowerfrequency sound bands with the most sustained effect being seen in the $0-250-\mathrm{Hz}$ band. The transient change in periodicity observed in this study is also in keeping with a soft palatal effect, as studies in animals and humans [6] have shown that the flapping tremor of the soft palate produces a complex, lowfrequency $(64-135 \mathrm{~Hz})$, highly periodic waveform. However, surgery did not significantly reduce the overall loudness of the snores that occurred, with no difference between the pre- and late post-operative data. The lack of effectiveness of either operation and lack of impact of risk factors, such as BMI or alcohol intake, on the results of surgery suggest that the lack of success relates to the structures targeted, rather than the selection of particular patient types to operate upon.

The current authors adopted a minimally invasive monitoring strategy, as many patients claim that sleeping in a polysomnography laboratory is not representative of their usual night's sleep. Establishing a reliable index that captures the number of snores in a "typical" night is difficult, since the quantity and character of snoring sound for any given individual is variable throughout sleep, between and within sleep stages and even within a single respiratory cycle $[7,26]$. Sleeping position has also been shown to alter the energy content of snores [27]. To minimise the impact of this variability and ensure that similar upper airway forces were involved in snore production, sound files, comprising the inspiratory component of snores whilst the patient was supine, were used for the acoustic analysis.

In conclusion, each year $>11,000$ people in the UK undergo surgery to alleviate snoring [33]. The evidence to support this practise is mostly based on retrospective reviews with shortterm follow-up, the few randomised prospective studies reported rely on subjectively reported outcomes [16]. Given the impracticality of offering sham surgery to overcome the inevitable placebo effect that accompanies such an intervention, it is particularly important to develop sensitive and objective markers of surgical effectiveness. The current study suggests that this is possible and that the analytical techniques used could identify changes in the relevant acoustic domains post-operatively. However, they also show that current surgery is largely ineffective in its goal of abolishing or significantly reducing snoring for a long period. The lack of a randomised observational cohort and the real possibility of regression to the mean in some of the changes observed make the current study the most optimistic estimate of the probable degree of success of this therapy. As such, it is difficult to recommend it for the routine treatment of socially disruptive snoring.

\section{ACKNOWLEDGEMENTS}

The authors would like to acknowledge the help of B. Cheetham (Dept of Computer Science, University of
Manchester, Manchester, UK) for his help in developing the acoustic measurements.

\section{REFERENCES}

1 Fairbanks DNF. Snoring: an overview with historical perspectives. In: Fairbanks DNF, Fujita $\mathrm{S}$, et al., eds. Snoring and obstructive sleep apnea. New York, Raven Press, 1987; pp. 1-18.

2 Ah-See KW, Stewart M, Banham SW, Robinson K, Carter R, Wilson JA. Systematic analysis of snoring in women. Ann Otol Rhinol Laryngol 1998; 107: 227-231.

3 Pelausa EO, Tarshis LM. Surgery for snoring. Laryngoscope 1989; 99: 1006-1010.

4 Fairbanks DN. Uvulopalatopharyngoplasty complications and avoidance strategies. Otolaryngol Head Neck Surg 1990; 102: 239-245.

5 Stradling JR, Crosby JH. Predictors and prevalence of obstructive sleep apnoea and snoring in 1001 middle aged men. Thorax 1991; 46: 85-90.

6 Beck R, Odeh M, Oliven A, Gavriely N. The acoustic properties of snores. Eur Respir J 1995; 8: 2120-2128.

7 Perez-Padilla JR, Slawinski E, Difrancesco LM, Feige RR, Remmers JE, Whitelaw WA. Characteristics of the snoring noise in patients with and without occlusive sleep apnea. Am Rev Respir Dis 1993; 147: 635-644.

8 Quinn SJ, Daly N, Ellis PD. Observation of the mechanism of snoring using sleep nasendoscopy. Clin Otolaryngol 1995; 20: 360-364.

9 Ikematsu T. Study of Snoring, 4th report: Therapy. J Jap Oto-rhino-laryngol 1964; 64: 434-435.

10 Fujita S, Conway W, Zorick F, Roth T. Surgical correction of anatomic abnormalities in obstructive sleep apnea syndrome: uvulopalatopharyngoplasty. Otolaryngol Head Neck Surg 1981; 89: 923-934.

11 Sharp JF, Jalaludin M, Murray JA, Maran AG. The uvulopalatopharyngoplasty operation: the Edinburgh experience. J R Soc Med 1990; 83: 569-570.

12 Koay CB, Freeland AP, Stradling JR. Short- and long-term outcomes of uvulopalatopharyngoplasty for snoring. Clin Otolaryngol 1995; 20: 45-48.

13 Croft CB, Golding-Wood DG. Uses and complications of uvulopalatopharyngoplasty. J Laryngol Otol 1990; 104: 871-875.

14 Ellis PD, Williams JE, Shneerson JM. Surgical relief of snoring due to palatal flutter: a preliminary report. Ann $R$ Coll Surg Engl 1993; 75: 286-290.

15 Wilde AD, Swift AC. A day-case procedure for treating simple snoring. Clin Otolaryngol 1995; 20: 486.

16 Jones TM, Ah-See K. Surgical and non-surgical interventions for non-apnoeic snoring patients. The Cochrane Library, issue 1. Chichester, John Wiley \& Sons Ltd, 2005.

17 Ellis PD, Williams JE, Shneerson JM. Surgical relief of snoring due to palatal flutter: a preliminary report. Ann $R$ Coll Surg Engl 1993; 75: 286-290.

18 Lynn P. Periodic signals. In: An introduction to the analysis and processing of signals. London, Macmillan Education Ltd, 1989; pp. 7-28.

19 Quinn SJ, Huang L, Ellis PD, Williams JE. The differentiation of snoring mechanisms using sound analysis. Clin Otolaryngol 1996; 21: 119-123. 
20 Agarwal S, Stone P, McGuinness K, Morris J, Camilleri AE. Sound frequency analysis and the site of snoring in natural and induced sleep. Clin Otolaryngol 2002; 27: 162-166.

21 Osborne JE, Osman EZ, Hill PD, Lee BV, Sparkes C. A new acoustic method of differentiating palatal from non-palatal snoring. Clin Otolaryngol 1999; 24: 130-133.

22 Young TB. Some methodologic and practical issues of reported snoring validity. Chest 1991; 99: 531-532.

23 Wiggins CL, Schmidt-Nowara WW, Coultas DB, Samet JM. Comparison of self- and spouse reports of snoring and other symptoms associated with sleep apnea syndrome. Sleep 1990; 13: 245-252.
24 Hoffstein V, Mateika S, Nash S. Comparing perceptions and measurements of snoring. Sleep 1996; 19: 783-789.

25 Gavriely N, Jensen O. Theory and measurements of snores. J Appl Physiol 1993; 74: 2828-2837.

26 Hill PD, Osman EZ, Osborne JE, Lee BW. Changes in snoring during natural sleep identified by acoustic crest factor analysis at different times of night. Clin Otolaryngol 2000; 25: 507-510.

27 Smithson AJ, White JE, Griffiths CJ, et al. Comparison of methods for assessing snoring. Clin Otolaryngol 1995; 20: 443-447. 